\title{
Indicações de transplante de córnea no Hospital de Clínicas de Porto Alegre
}

\author{
Indications for corneal graft surgery at the Hospital de Clínicas of Porto Alegre
}

\author{
Silvana Cattani ${ }^{1}$ \\ Sergio Kwitko ${ }^{2}$ \\ Marco Antonio Hermann Kroeff ${ }^{3}$ \\ Diane Marinho ${ }^{4}$ \\ Samuel Rymer ${ }^{5}$ \\ Francisco de Lima Bocaccio ${ }^{6}$
}

\section{RESUMO}

Objetivo: Definir as indicações mais freqüentes de ceratoplastia penetrante no Serviço de Oftalmologia da Universidade Federal do Rio Grande do Sul - a verificação da doença corneana mais transplantada bem como as médias etária e de tempo de preservação das córneas utilizadas. Métodos: Foi realizado um estudo retrospectivo comparativo entre os pacientes atendidos e que tiveram indicação de transplante de córnea (transplantados e em lista de espera) no período de janeiro de 1988 a dezembro de 1997. Resultado: As principais indicações foram: ceratocone $(28,6 \%)$, ceratopatia bolhosa $(20,9 \%)$, leucoma $(12,6 \%)$ e retransplante $(11,0 \%)$. A doença mais transplantada foi o ceratocone (95\% dos casos). As médias etária e de preservação das córneas utilizadas foram 44 anos e 6,6 dias respectivamente. Conclusão: As principais indicações de ceratoplastia penetratnte em nosso meio são o ceratocone, a ceratopatia bolhosa, os leucomas e o retransplante. A doença mais transplantada foi o ceratocone por ser a doença mais incidente e não pelo fato de termos maior oferta de córneas doadoras idosas como suspeitávamos inicialmente. Quanto às médias etária e de tempo de preservação das córneas utilizadas houve preferência estatisticamente significativa pelo uso de córneas mais jovens nos casos de ceratopatia bolhosa.

Descritores: Transplante de córnea; Doadores de tecidos; Ceratoplastia penetrante; Preservação de órgãos; Estudos retrospectivos

Trabalho realizado no Serviço de Oftalmologia do Hospital de Clínicas de Porto Alegre.

${ }^{1}$ Médica Oftalmologista colaboradora do Serviço de Oftalmologia do Hospital de Clínicas de Porto Alegre.

${ }^{2}$ Médico do Setor de Córnea do Serviço de Oftalmologia do Hospital de Clínicas de Porto Alegre. Doutor em Oftalmologia.

${ }^{3}$ Médico Residente do Serviço de Oftalmologia do Hospital de Clínicas de Porto Alegre.

${ }^{4}$ Médica do Setor de Córnea do Serviço de Oftalmologia do Hospital de Clínicas de Porto Alegre. Mestre em Oftalmologia.

${ }^{5}$ Chefe do Setor de Córnea do Hospital de Clínicas de Porto Alegre. Professor Adjunto da Disciplina de Oftalmologia da Universidade Federal do Rio Grande do Sul. Mestre em Oftalmologia.

${ }^{6}$ Médico do Setor de Córnea do Hospital das Clínicas de Porto Alegre. Professor Assistente da Disciplina de Oftalmologia da Universidade Federal do Rio Grande do Sul.

Endereço para correspondência: Av. Goethe, 21-sala 504 - Porto Alegre (RS) CEP 90040-100.

E-mail: scattani@terra.com.br

Recebido para publicação em 11.11.2000

Aceito para publicação em 02.07.2001

\section{INTRODUÇ̃̃O}

\begin{tabular}{l} 
INTRODUÇÃo \\
\hline A indicação de ceratoplastia penetrante tem aumentado muito nos últimos \\
30 anos e, atualmente, está entre as cirurgias de transplante mais realizadas ${ }^{(1-3)}$. \\
O aumento na indicação de ceratoplastia penetrante se deve a alguns fatores, \\
entre eles o aumento da faixa etária da população, melhor seleção do tecido \\
doador e novas técnicas operatórias que permitem a realização de cirurgias em \\
situações consideradas inoperáveis até 10 anos atrás ${ }^{(4)}$. \\
As estatísticas indicam que, apenas nos Estados Unidos da América \\
(EUA), são realizadas mais de 35.000 ceratoplastias anualmente ${ }^{(4)}$. A indica- \\
ção mais comum varia muito quando analisados vários estudos, dependen- \\
do do período de tempo estudado, do método de coleta de dados, do local \\
de obtenção dos dados e da população base de pacientes do cirurgião. \\
Nos EUA a principal indicação de ceratoplastia penetrante é a ceratopa- \\
tia bolhosa, diferindo da Europa e do Brasil, onde a maioria dos relatos \\
mostram o ceratocone como a indicação mais freqüente ${ }^{(5-7)}$. Comparando-se \\
os resultados obtidos pelos vários relatos de diferentes países, questiona- \\
mos se o ceratocone é realmente a principal indicação por ser a condição
\end{tabular}


mais freqüente entre as indicadas, ou se isso se deve ao fato de termos um maior número de doações de córnea entre pacientes de uma faixa etária mais avançada.

Motivados por esta dúvida, consideramos relevante fazer uma análise retrospectiva das causas de transplante de córnea no Hospital de Clínicas de Porto Alegre (HCPA), tendo como objetivos: (1) definir as indicações mais freqüentes de transplante de córnea em nosso meio; (2) verificar a patologia corneana mais freqüentemente transplantada; (3) verificar as médias etárias e de tempo de preservação das córneas doadoras utilizadas em nosso Serviço.

\section{MÉTODOS}

Realizou-se um estudo retrospectivo comparativo entre todos os pacientes atendidos no serviço de oftalmologia do HCPA, que tiveram indicação de transplante de córnea (transplantados e em lista de espera) no período entre janeiro de 1988 e dezembro de 1997. Foram avaliados o diagnóstico corneano na indicação do transplante nos pacientes que aguardavam na lista de espera e nos pacientes já transplantados. Foram avaliados também a idade e tempo de preservação das córneas doadoras utilizadas. Os dados foram armazenados em um banco de dados e analisados estatisticamente pelo teste t-student.

\section{RESULTADOS}

Durante o período analisado, foram incluídos na lista de espera para ceratoplastia penetrante um total de 507 olhos, cujas indicações estão relacionadas na tabela 1. Observa-se que o ceratocone e a ceratopatia bolhosa foram as indicações mais freqüientes.

Do total de 507 casos colocados em lista de espera, 454 foram operados no período do presente estudo. Dos 145 casos de ceratocone, foram submetidos a cirurgia 138 (95\%). Quanto a ceratopatia bolhosa, dos 106 casos, $83(88 \%)$ foram submetidos à ceratoplastia. Portanto, o ceratocone foi a doença corneana mais submetida à ceratoplastia penetrante tanto em números absolutos como em números relativos. A tabela 2 apresenta as indicações dos casos operados no mesmo período.

Tabela 1. Principais indicações de transplante de córnea no HCPA (lista de espera de jan/88 a dez/97)

\begin{tabular}{lcc} 
Indicação & № de casos & $\%$ \\
Ceratocone & 145 & $28,6 \%$ \\
Ceratopatia bolhosa & 106 & $20,9 \%$ \\
Leucoma corneano & 64 & $12,6 \%$ \\
Retransplante & 56 & $11,0 \%$ \\
Úlceras não herpéticas & 22 & $4,3 \%$ \\
Distrofia de Fuchs & 19 & $3,8 \%$ \\
Herpes simples & 17 & $3,4 \%$ \\
Outros & 78 & $15,4 \%$ \\
Total & $\mathbf{5 0 7}$ & $\mathbf{1 0 0} \%$ \\
\hline
\end{tabular}

\begin{tabular}{|lcc|}
\hline \multicolumn{3}{|c|}{ Tabela 2. Principais indicações dos transplantes realizados no } \\
HCPA (jan/88 a dez/97) \\
Indicação & Casos operados & $\%$ \\
Ceratocone & 138 & $30,4 \%$ \\
Ceratopatia bolhosa & 83 & $18,3 \%$ \\
Leucoma corneano & 60 & $13,2 \%$ \\
Retransplante & 53 & $11,7 \%$ \\
Úlceras não herpéticas & 22 & $4,8 \%$ \\
Distrofia de Fuchs & 18 & $4 \%$ \\
Herpes simples & 16 & $3,5 \%$ \\
Outros & 64 & $14,1 \%$ \\
Total & $\mathbf{4 5 4}$ & $\mathbf{1 0 0} \%$ \\
\hline
\end{tabular}

A idade média das córneas doadoras utilizadas nos 454 transplantes realizados, foi 44 anos (mínimo de 3 anos e máximo de 86 anos). O tempo médio de preservação destas córneas foi de 6,6 dias (mínimo de 1 dia e máximo de 19 dias).

Em relação às duas principais indicações de transplante, ceratocone e ceratopatia bolhosa, o tempo médio de preservação das córneas utilizadas nesses casos foi de 7,3 dias para a primeira e 6,8 dias para a segunda. A idade média das córneas doadoras utilizadas nos casos de ceratocone e ceratopatia bolhosa (48,7\% dos casos) foi de 60 anos e 48 anos respectivamente. Esta diferença foi estatisticamente significativa $(\mathrm{p}<0,0001)$, mostrando que houve uma preferência pelo uso de córneas mais jovens em casos de ceratopatia bolhosa. As córneas utilizadas nos demais transplantes (51,3\% dos casos) tiveram idade média de 33,5 anos.

\section{DISCUSSÃO}

Nosso estudo corrobora os achados da literatura no que se refere às alterações na frequência das ceratopatias com relação à ceratoplastia penetrante (tabela 3). As principais indicações de ceratoplastia penetrante têm sido modificadas ao longo dos últimos 30 anos. Segundo autores americanos, até 1970 as causas mais freqüientes eram os leucomas herpéticos, os retransplantes e os ceratocones. De 1970 até 1980 foram diminuindo as indicações devidas ao herpes, em conseqüência do aparecimento de novos fármacos e tratamentos mais eficazes, e aumentando o número de transplantes por ceratopatia bolhosa, permanecendo quase inalterado o número de casos de ceratocone e outras indicações ${ }^{(1)}$. Desde 1980 até os dias de hoje, observou-se um aumento no número de indicações por ceratopatia bolhosa, superando as demais indicações de transplante nos EUA. Lindquist ${ }^{(4)}$, realizou um estudo em 1988, onde encontrou como causas principais de ceratoplastia penetrante a ceratopatia bolhosa $(26,7 \%)$ e o ceratocone $(17,6 \%)$, seguidos pelo leucoma com ou sem inflamação $(14,9 \%)$, retransplante $(10,7 \%)$, distrofia de Fuchs $(10,7 \%)$, ceratite herpética $(5,4 \%)$ ceratites ulceradas $(4,2 \%)$ e traumatismos $(2,3 \%)$. Lois ${ }^{(8)}$, em um estudo retrospectivo de 2442 casos de transplante de córnea realizados no período entre janeiro/1989 e dezembro/1995, encontrou como princi- 


\begin{tabular}{|c|c|c|c|c|c|c|}
\hline Indicações & $\begin{array}{c}\text { HCPA } \\
\text { (Brasil) }\end{array}$ & $\begin{array}{l}\text { Trindade } \\
\text { (Brasil) }\end{array}$ & $\begin{array}{l}\text { Kervick } \\
\text { (Irlanda) }\end{array}$ & $\begin{array}{c}\text { Acedo } \\
\text { (Espanha) }\end{array}$ & $\begin{array}{l}\text { Lindquist } \\
\text { (EUA) }\end{array}$ & $\begin{array}{l}\text { Lois } \\
\text { (EUA) }\end{array}$ \\
\hline $1^{\circ}$ & $\begin{array}{c}\text { Ceratocone } \\
(28,6 \%)\end{array}$ & $\begin{array}{c}\text { Ceratocone } \\
(24 \%)\end{array}$ & $\begin{array}{c}\text { Ceratocone } \\
(28,57 \%)\end{array}$ & $\begin{array}{c}\text { Ceratocone } \\
(28,97 \%)\end{array}$ & $\begin{array}{c}\text { C. bolhosa } \\
(26,7 \%)\end{array}$ & $\begin{array}{c}\text { C.Bolhosa } \\
(26 \%)\end{array}$ \\
\hline $2^{\circ}$ & $\begin{array}{c}\text { C.Bolhosa } \\
(20,9 \%)\end{array}$ & $\begin{array}{l}\text { Leucoma } \\
(22 \%)\end{array}$ & $\begin{array}{l}\text { C.Herpes } \\
(23,42 \%)\end{array}$ & $\begin{array}{l}\text { Leucoma } \\
(21,87 \%)\end{array}$ & $\begin{array}{c}\text { Ceratocone } \\
(17,6 \%)\end{array}$ & $\begin{array}{c}\text { Retransplante } \\
\quad(17,8 \%)\end{array}$ \\
\hline $3^{\circ}$ & $\begin{array}{c}\text { Leucoma } \\
(12,6 \%)\end{array}$ & $\begin{array}{l}\text { Falência } \\
(20 \%)\end{array}$ & $\begin{array}{l}\text { D.Fuchs } \\
(9,71 \%)\end{array}$ & $\begin{array}{c}\text { C.Bolhosa } \\
(9,20 \%)\end{array}$ & $\begin{array}{c}\text { Leucomas } \\
(14,9 \%)\end{array}$ & $\begin{array}{l}\text { D.Duchs } \\
(15,7 \%)\end{array}$ \\
\hline $4^{\circ}$ & $\begin{array}{c}\text { Retransplante } \\
(11,0 \%)\end{array}$ & $\begin{array}{c}\text { C.Bolhosa } \\
(16 \%)\end{array}$ & $\begin{array}{l}\text { Falência } \\
(9,14 \%)\end{array}$ & $\begin{array}{c}\text { Combinada } \\
(7,95 \%)\end{array}$ & $\begin{array}{l}\text { Falência } \\
(10,7 \%)\end{array}$ & $\begin{array}{c}\text { Ceratocone } \\
(13,2 \%)\end{array}$ \\
\hline
\end{tabular}

pais indicações: bolhosa pós facectomia (26\%), retransplante (17,8\%), distrofia de Fuchs $(15,7 \%)$ e ceratocone $(13,2 \%)$. No entanto, o autor salienta que do total de casos de bolhosa pós facectomia, 54,7\% estavam associados com lentes de câmara anterior. Outra série de 1104 casos de ceratoplastia penetrante publicada por Flowers ${ }^{(9)}$ também mostra a ceratoplastia bolhosa como principal indicação, mas os autores comentam que esta indicação foi decrescendo ao longo do período do estudo. Entretanto, em estudos europeus ${ }^{(1,10)}$, o que se observou foi uma casuística um pouco diferente, com ceratocone como principal indicação.

Uma possível explicação para esta diferença, entre a incidência de ceratopatia bolhosa nos EUA e na Europa, pode ser o fato de que as primeiras lentes intra-oculares utilizadas nos EUA tenham sido lentes rígidas de câmara anterior e de fixação iriana. Estas provocaram uma descompensação corneana, o que fez com que a ceratopatia bolhosa fosse a maior indicação de transplante nos EUA nas duas últimas décadas. O mesmo não ocorreu na Europa e na América Latina, onde as lentes referidas foram pouco utilizadas ${ }^{(1,4)}$.

Em estudos brasileiros ${ }^{(5-7)}$ a principal indicação de ceratoplastia penetrante é o ceratocone, seguido da ceratopatia bolhosa. Segundo Thomaz ${ }^{(6)}$, a maior incidência de ceratoplastias por ceratocone poderia ser explicada pelos ótimos resultados que se obtém com esse tratamento.

Em nossos 507 casos com indicação de transplante, as mais freqüentes foram: ceratocone $(28,6 \%)$, ceratopatia bolhosa $(20,9 \%)$, leucoma $(12,6 \%)$ e retransplante $(11,0 \%)$. Estes dados são bastante semelhantes aos encontrados em outro estudo brasileiro realizado por Gonçalves e Trindade ${ }^{(5)}$, que também encontrou como indicações mais freqüentes, o ceratocone (24\%), leucoma (22\%), retransplante (20\%) e ceratopatia bolhosa $(16 \%)$.

No relato de Pacheco ${ }^{(11)}$ a principal indicação foi a ceratoplastia bolhosa da afacia, sendo o ceratocone a terceira indicação. Segundo os autores, esse declínio na incidência de ceratocone deve-se à melhora nas técnicas de adaptação e fabricação de lentes de contato. Esse dado difere do encontrado em nosso estudo e no restante da bibliografia brasileira consultada.

Podemos observar que, de um modo geral, as casuísticas citadas se assemelham à nossa. As discordâncias encontra- das podem se dever às diferenças quanto à atualidade dos artigos, à seletividade dos pacientes atendidos em cada Serviço, bem como ao aprimoramento no tratamento clínico e cirúrgico das doenças corneanas. Mesmo os trabalhos que apontam a ceratopatia bolhosa como principal indicação, verificam que esta vem diminuindo ao longo dos anos. Fatores como o aprimoramento da técnica de facectomia, melhora da qualidade das lentes intra-oculares e o maior uso de substâncias viscoelásticas protetoras do endotélio corneano, explicam o decréscimo de ceratoplastias por essa condição ${ }^{(6)}$.

Com relação à idade média das córneas doadoras, houve uma preferência pelo uso de córneas mais jovens nos casos de ceratopatia bolhosa (48 anos) em relação às utilizadas nos casos de ceratocone (60 anos). Essa preferência, provavelmente, deveu-se ao fato de a ceratopatia bolhosa, segundo vários autores $^{(1,8,12-14)}$, ter um índice de sucesso pós transplante menor do que o ceratocone. Pacientes com ceratopatia bolhosa possuem um endotélio corneano anormal ${ }^{(15)}$ necessitando, portanto, de córneas doadoras mais jovens, as quais têm uma maior contagem endotelial. Como os doadores com mais de 60 anos são mais freqüentes (córneas com menor contagem endotelial), selecionou-se pacientes portadores de ceratocone para estas córneas, por possuírem um endotélio corneano normal. Isso justifica o maior número de transplantes nos casos de ceratocone.

\section{CONCLUSÃO}

Os resultados do nosso estudo nos permitem concluir que as principais indicações de ceratoplastia penetrante, em nosso meio, são o ceratocone $(28,6 \%)$, a ceratopatia bolhosa $(20,9 \%)$, os leucomas $(12,6 \%)$ e o retransplante $(11,0 \%)$. Estes achados, de um modo geral, são compatíveis com os descritos na literatura. A patologia mais transplantada foi o ceratocone, tendo em vista a maior oferta de córneas doadoras com menor contagem endotelial. Quanto às médias etária e de tempo de preservação das córneas utilizadas, foram 44 anos e 6,6 dias respectivamente. Houve preferência estatisticamente significativa pelo uso de córneas mais jovens nos casos de ceratopatia bolhosa. 
Pensávamos inicialmente que o maior número de casos de ceratocone transplantados se devesse apenas à maior oferta de córneas doadoras idosas. Na realidade comprovamos que, em nosso Serviço, existem mais casos de ceratocone tanto entre os transplantados como na lista de espera.

\section{ABSTRACT}

Purpose: To evaluate the most frequent indications for penetrating keratoplasty $(\mathrm{PK})$ in the Ophthalmology service of the Hospital de Clínicas of Porto Alegre. Methods: Retrospective analysis of the charts of 507 patients with indication for penetrating keratoplasty during the period of Jan/1988 to Dec/ 1997 (454 underwent the surgery during the period). Results: The main indications for PK were as follows: keratoconus (28.6\%), bullous keratopathy (20.9\%), leucomas (12.6\%) and regraft $(11.0 \%)$. The age average of the corneas was 44 years and the mean preservation time was 6,6 days. Conclusions: There was agreement between our study and the literature despite some differences between the main indications.

Keywords: Corneal transplantation; Tissue donors; Keratoplasty/penetrating; Organ preservation; Retrospective studies

\section{REFERÊNCIAS}

1. Acedo JT. Queratoplastias y queratoprotesis. Barcelona: EdikaMed; 1992.

2. Brightbill FS. Keratoplasty. In: Brightbill FS. Corneal surgery. Theory, technique and tissue. Saint Louis: C. V. Mosby; 1986. p.32-58.

3. Belfort Jr R. Ceratoplastias e ceratectomia. In: Belfort Jr R, Kara-Jose N Córnea. São Paulo: Roca; 1996. p.493-504.

4. Lindquist TD, McGlothan JS, Rotkis WM, Chandler JW. Indications for penetrating Keratoplasty: 1980-1988. Cornea 1991;10:210-6.

5. Gonçalves EC, Trindade FC. Ceratoplastia penetrante: alterações nas indicações, 1983-1992. Arch Bras Oftalmol 1994;53:274-7.

6. Thomaz A, Ando E, Akaishi L, Barros A. Rejeição em transplante de córnea. Rev Bras Oftalmol 1990;49:15-20.

7. Barros CR, Oliveira DF, Castro RS, Lima VMP, Kara José N. Incidência e falência em transplante de córnea. Arq Bras Oftalmol 1992;55:176-81.

8. Lois N, Kowal VO, Cohen EJ, Rapuano CJ, Gault JA, Raber IM, Laibson PR. Indications for penetrating keratoplasty and associated procedures, 19891995. Córnea 1997;16:623-9.

9. Flowers CW, Chanq KY, McLeod SD, Irvine JA, McDonnell PJ, Rao N, et al. Changing indications for penetrating keratoplasty, 1989-1993. [comment on Cornea 1997;16:120-1]. Cornea 1995;14:583-8.

10. Kervick GN, Shepherd WF. Changing indications for penetrating keratoplasty (brief notes). Ophtalmic Surg 1990;21:227.

11. Pacheco LFR, Pena AS, Florence M. Indicações para ceratoplastia penetrante Arq Bras Oftalmol 1990;53:163-6.

12. Forster RK, Fine M. Relation of donnor age to success in penetrating keratoplasty. Arch Ophtalmol 1971;85:42-7.

13. Jenkins MS, Lempert SL, Brown SI. Significance of donnor age in penetrating keratoplasty. Ann Ophtalmol 1979;11:974-6.

14. Polack FM. Influence of host corneal disease in the prognosis of keratoplasty. In: Polack FM. Corneal transplantation. New York: Grune \& Stratton; 1977. p.163-75

15. Shaw EL, Rao GN, Arthur EJ, Aquavella JV. The functional reserve of the corneal endotelium. Ophthalmology 1978;85:640-9.

\section{CONGRESSO BRASILERO DE PREVENCÄO DA CEGUERA E REABLITACẠAO VISUAL}

\section{1 de Agosto a 3 de Setembro de 2002 - Curitiba - PR PARTICIPEM}

TAXAS DE INSCRIC̣ÃO (VALORES EM REAIS - R\$)

\section{Categoria}

Sócios do CBO

Não Sócios do CBO

Residentes e Acadêmicos

Outros profissionais da área de saúde

Auxiliares em Oftalmologia

Acompanhantes

\section{Cursos Pagos}

Inscrição por Curso

$\mathbf{3 0 / 0 4 / 0 2}$
$R \$ 190,00$
$R \$ 420,00$
$R \$ \quad 95,00$
$R \$ \quad 95,00$
$R \$ \quad 50,00$
$R \$ \quad 50,00$

15/06/02

$R \$ 250,00$

$R \$ 480,00$

$R \$ 125,00$

$R \$ 125,00$

$R \$ 65,00$

$R \$ 65,00$

$\begin{array}{lr}\mathbf{3 0 / 0 7 / 0 2} \\ R \$ & 300,00 \\ R \$ & 530,00 \\ R \$ & 150,00 \\ R \$ & 150,00 \\ R \$ & 80,00 \\ R \$ & 80,00\end{array}$

No local

$R \$ 400,00$

$R \$ \quad 625,00$

$R \$ 200,00$

$R \$ 200,00$

$R \$ \quad 95,00$

$R \$ \quad 95,00$

\section{INFORMAC̣ÕES: Conselho Brasileiro de Oftalmologia CBO-Eventos: Fone (11) 3266-4000 - Fax: $3171-0948$ - e-mail: eventos@cbo.com.br}

